\title{
Enabling Sector Scheduling for 5G-CPE Dense Networks
}

\author{
Jie Yang $\left(\mathbb{D}\right.$, Li Feng $\mathbb{D}^{\mathbb{D}}$, Tong Jin, Hong Liang $\mathbb{D}^{\mathbb{D}}$, Fangxin Xu $\mathbb{D}$, and Liwei Tian \\ Faculty of Information Technology, Macau University of Science and Technology, Taipa, Macau 999078, China \\ Correspondence should be addressed to Li Feng; lfeng@must.edu.mo
}

Received 15 October 2020; Revised 23 November 2020; Accepted 8 December 2020; Published 28 December 2020

Academic Editor: Lingwei Xu

Copyright (C) 2020 Jie Yang et al. This is an open access article distributed under the Creative Commons Attribution License, which permits unrestricted use, distribution, and reproduction in any medium, provided the original work is properly cited.

$5 \mathrm{G}$ customer premise equipment (5G-CPE) is an IoT gateway technology that integrates $5 \mathrm{G}$ and Wi-Fi and therefore can provide Wi-Fi connection for IoT devices and meanwhile benefit from the advantages of 5G. With the increasing number of IoT devices, transmission collisions and hidden/exposed terminal problems on the Wi-Fi connection side become more and more serious. Conventional mechanisms cannot solve these problems well. In this paper, we propose a Wi-Fi sector (Wi-FiS) design, which is compatible with Wi-Fi, to solve them fundamentally. Wi-FiS divides the whole coverage area of Wi-Fi into multiple sectors and utilizes beamforming technology and sector-based scheduling to improve system performance of Wi-Fi dense networks. For a single-cell network, Wi-FiS differentiates uplink and downlink operations and totally excludes collision in downlink. For a multicell network, Wi-FiS can avoid hidden and exposed terminal problems, while enabling parallel transmissions among multiple cells. We then develop a theoretical model to analyze Wi-FiS's throughput. Extensive simulations verify that our theoretical model is very accurate and Wi-FiS can improve system throughput of Wi-Fi dense networks significantly.

\section{Introduction}

In Industry 4.0, a great many Internet of Things (IoT) devices are widely deployed in multiple factories. In each factory, IoT devices work together to coordinate and monitor progress along assembly lines, under the control of an operation control center. Usually, the operation control center is located in a cloud or an edge server. To achieve realtime control, Industry 4.0 requires a high-speed and lowlatency network between the operation control center and these IoT devices.

$5 \mathrm{G}$ technology is promising to meet the network requirement of Industry 4.0, since it can provide a high speed of $10 \mathrm{Gbps}$ and a low latency of $1 \mathrm{~ms}-10 \mathrm{~ms}$ [1-3]. To apply $5 \mathrm{G}$ technology to Industry 4.0, we need to establish a $5 \mathrm{G}$ base station which connects these IoT devices and the operation control center, and it is required that these IoT devices are $5 \mathrm{G}$ enabled. However, to date, deploying $5 \mathrm{G}$ base stations is expensive, and 5G modules, which are large in size and power-consuming, are therefore not suitable to install in resource-limited IoT devices.

To overcome the above problem, we may adopt 5G customer premise equipment (5G-CPE) solution. In this solution, 5G-CPE, a 5G- and Wi-Fi-enabled node, is introduced to act as a relay between IoT devices and the $5 \mathrm{G}$ base station, as shown in Figure 1. The advantages of this solution are twofold. On the one hand, most of IoT devices support Wi-Fi due to low-cost Wi-Fi modules; however, it is generally expensive and difficult to deploy wired network infrastructure such as optical fibers to connect Wi-Fi base stations, due to constraint of factories' geographical locations. Therefore, enabling 5G-CPE to connect IoT devices via $\mathrm{Wi}-\mathrm{Fi}$ is convenient, economic, and fast. On the other hand, utilizing 5G-CPE to connect the operation control center via $5 \mathrm{G}$ may fully take advantage of $5 \mathrm{G}$ 's high bandwidth and low latency.

1.1. Motivation. In this paper, for the 5G-CPE solution, we are concerned with the 5G-CPE network, i.e., the Wi-Fi network where IoT devices communicate with the 5G-CPE. As the number of IoT devices grows, the channel congestion, transmission collision, and hidden/exposed terminal problems of 5G-CPE network will become more and more serious. Usually, there are two methods to solve these problems in large-scale Wi-Fi dense networks; however, the 


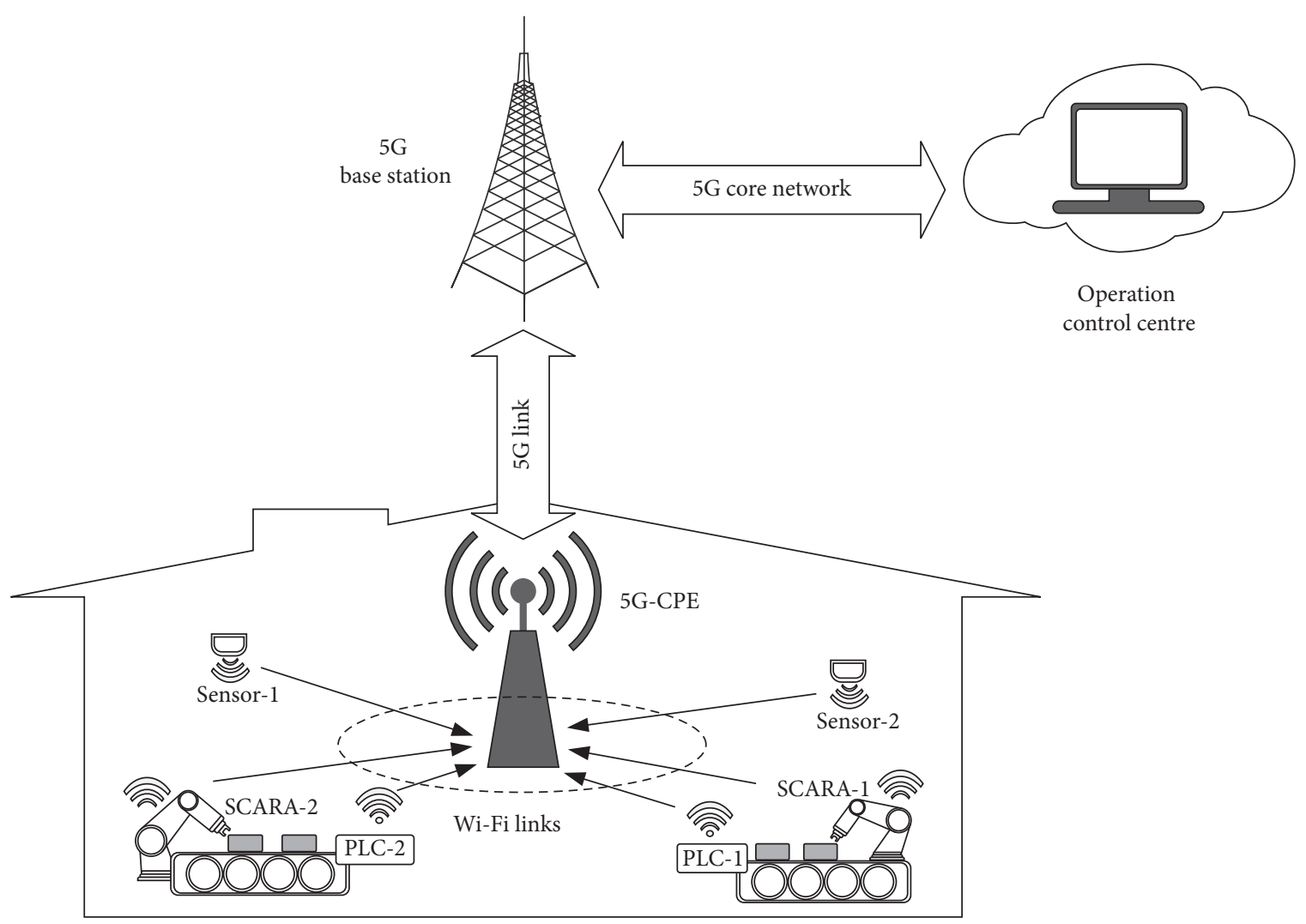

Figure 1: 5G-CPE for Industry 4.0.

two methods only achieve limited performance improvement and therefore do not solve these problems fundamentally [4-8].

(i) The first one is the contention window- (CW-) based adjustment method. In $\mathrm{Wi}-\mathrm{Fi}$, each node chooses a random time between 0 and $\mathrm{CW}$ as a waiting time before data transmission. In this method, as the number of nodes grows, the CW size increases and therefore the average waiting time of each node will increase. A longer waiting time will help reduce the collision probability. However, a longer waiting time will lead to much time waste in channel contention.

(ii) The second one is the request-to-send/clear-to-send (RTS/CTS) method. In this method, by sending short RTS frames to reserve channel, nodes can reduce the time waste caused by the collision of large data. However, this method introduces additional transmission times of RTS and CTS frames and a large population of nodes will still lead to serious RTS collision.

In addition, for multicell networks, existing methods cannot well solve the hidden and exposed terminal problems. For example, the RTS/CTS method solves the hidden terminal problem, but it also induces the exposed terminal problem simultaneously [6-8]. These challenges motivate this study.
1.2. Our Contributions. In this paper, we propose a Wi-Fi sector (for short, Wi-FiS) design, which is compatible with $\mathrm{Wi}-\mathrm{Fi}$, to solve the aforementioned challenges fundamentally. Wi-FiS borrows the hard-disk sector idea [9] to divide the whole coverage area of a 5G-CPE dense network into multiple sectors (as shown in Figure 2), each sector including a small number of nodes. Hereafter, we use the access point (AP) to represent the 5G-CPE for shortening notations. Benefitting beamforming technology and sectorbased scheduling, Wi-FiS can improve the system performance of single- and multicell networks significantly. Our contributions are summarized as follows.

(i) For a single-cell network, Wi-FiS groups contention nodes and performs sector scheduling. With WiFiS, the access point (AP) first silences all nodes and then activates each sector sequentially via directional beam. In this way, while keeping compatibility with $\mathrm{Wi}-\mathrm{Fi}$, Wi-FiS can reduce contention collision and improve system throughput of dense networks significantly.

(ii) For a multicell network, Wi-FiS can avoid hidden and exposed terminal problems, while enabling parallel transmissions among multiple cells. With $\mathrm{Wi}-\mathrm{FiS}$, once detecting one ongoing transmission in one cell that might lead to a potential hidden/exposed terminal problem, the AP in another cell may 


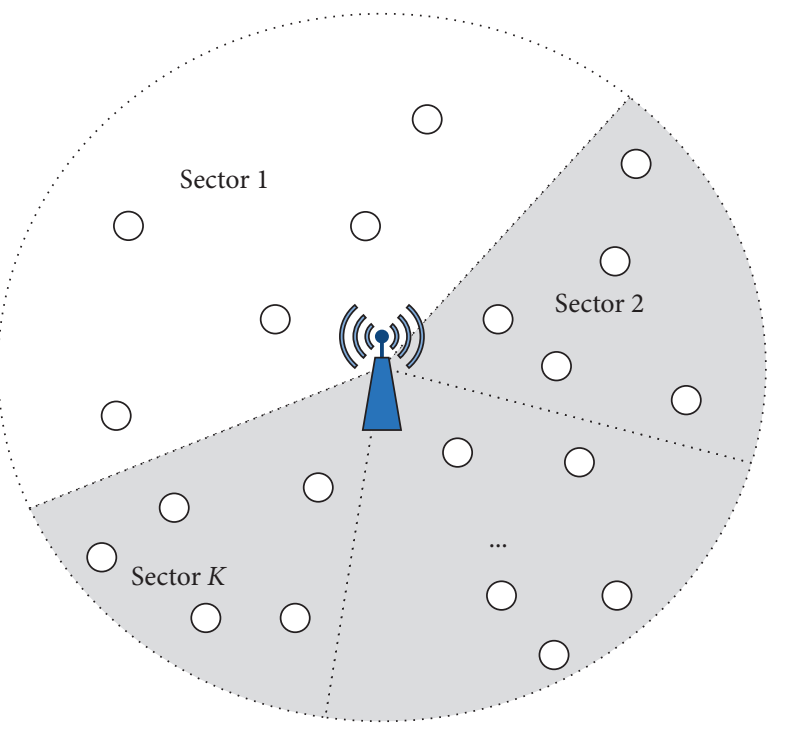

FIgURE 2: Software-defined MAC for sector scheduling.

trigger its directional transmissions without interfering with the ongoing transmission.

(iii) We run extensive simulations to verify that our WiFiS design is very effective and can significantly outperform conventional methods in terms of system throughput.

The rest of the paper is organized as follows. Section 2 summarizes related works. Section 3 presents the Wi-FiS architecture. Sections 4 and 5 present Wi-FiS scheduling for single- and multicell, respectively. Section 6 analyzes $\mathrm{Wi}$ FiS's throughput theoretically. Section 7 evaluates Wi-FiS's performance. Finally, Section 8 concludes this paper.

\section{Related Works}

Currently, there are mainly two types of methods that solve the collision problem of large-scale networks [4-14]: dynamic CW adjustment and RTS/CTS-based methods. These two methods and their shortcomings are briefly described below.

(i) Dynamic CW adjustment method: this method reduces transmission collision via contention window adjustment.

(1) Shahin et al., Deng et al., and Ali et al. [10-12] studied the collision problem in single-cell scenario. Shahin et al. [10] used a channel observation-based mechanism to monitor the real-time collision probability of the channel and implemented dynamic adjustment of the contention window accordingly, avoiding the high delay caused by blindly multiplying the contention window. Deng et al. [11] proposed a bit-error-ratio channel adjustment algorithm to design the contention window under the dense network to obtain higher performance. Ali et al. [12] introduced a reinforcement learning method to observe the channel state and selected the optimal contention window accordingly. However, these methods still lead to large contention overhead in dense network environments.

(2) Sen et al. $[4,5]$ studied the hidden terminal problems in multicell scenario. CSMA/CN [4] solved the hidden terminal problem by detecting collision notification information. Gollakota and Katabi [5] proposed a method to decode the collision packets when a hidden terminal problem occurs. However, these methods can only solve the hidden terminal problem, but cannot combat the exposed terminal problem.

(ii) RTS/CTS-based methods: this method reduces the collision via RTS/CTS-based channel reservation.

(1) Wang et al. $[13,14]$ proposed a RTS/CTS method to solve the collision problem in single-cell scenario, where nodes will send an RTS frame before its data transmission. This introduces additional control overhead of RTS/CTS.

(2) Xiong et al. [6-8] studied the hidden/exposed problems in multicell scenario. Xiong et al. [6] designed a novel S-CTS packet, which can be detected in lower SNR (signal to noise) environments, to combat the hidden terminal problem. Magistretti et al. [7] exploited crosscorrelation to detect control frames (such as RTS, CTS, and ACK); this design can tolerate strong interferences in multicell environments. MACA-P [8] utilized RTS/CTS handshakes to schedule the node-pairs' transmission order, which can help avoid the hidden terminal problem and increase transmission concurrency. However, these methods cannot resolve the traditional exposed terminal problem and will lead to a new NAV type exposed terminal problem.

Different from the above two types of research studies, our design adopts beamforming and NAV mechanisms to solve the collision and hidden/exposed terminal problems in an entire network. Particularly, using virtual carrier sense, our design can only activate parts of nodes each time for contention and therefore can reduce the collision probability significantly.

\section{Wi-FiS Architecture}

In this section, we describe the architecture design of Wi-FiS.

We design Wi-FiS based on the standard Wi-Fi framework [15]. Below, we illustrate the MAC design and PHY design of Wi-FiS (as shown in Figure 3):

(i) Wi-FiS PHY: based on standard Wi-Fi PHY layer, Wi-FiS introduces sector-beam transmission technology. Using this technology, AP can transmit and control specific physical sector areas. 


\begin{tabular}{c|c|}
\cline { 2 - 2 } Wi-FiS MAC & $\begin{array}{c}\text { Wi-Fi MAC } \\
\text { (supporting sector scheduling) }\end{array}$ \\
\cline { 2 - 2 } Wi-FiS PHY & $\begin{array}{c}\text { Wi-Fi PHY } \\
\text { (supporting sector beam) }\end{array}$ \\
\cline { 2 - 2 }
\end{tabular}

Figure 3: Wi-FiS architecture.

(ii) Wi-FiS MAC: based on standard Wi-Fi MAC layer, Wi-FiS introduces the sector-scheduling technology based on sector. Using this technology, the AP can trigger each sector one by one.

3.1. PHY Design. Here, we present the hardware of the AP that supports multiple antennas for directional beamforming.

Based on hardware support, our design can improve the 802.11 ac version or newer routing protocols, as shown in Figure 4. 802.11ac routing already supports using up to 8 antennas in a single channel to form beams, so it can provide directional beams. In our design, Wi-FiS can be installed directly on $802.11 \mathrm{ac}$ devices (including the 5G-CPE or 802.11 ac router) without hardware modification. We assume that AP or STAs have up to 8 antennas, so that they can perform beamforming under a single channel, and these antennas are all omnidirectional antennas. In particular, these 8 antennas can provide beamforming technology in the form of digital precoding according to 802.11 protocol standard to achieve directional scanning.

In this paper, we adopt DOA-based location detection technology and RSSI power detection technology [16-18] for the physical layer, and we assume that the default transmission power of all nodes is the same and known (the power is negotiated in during the association process). DOA and RSSI functionalities can be implemented by updating the firmware of software. Therefore, in our design, AP can get the relative position (including relative angle and distance) of nodes around it via the DOA and RSSI technologies.

3.2. MAC Design. In this section, we present MAC design of Wi-FiS. Wi-FiS divides the whole network coverage into sectors and then activates/schedules its sectors sequentially via its PHY and MAC technologies. Below, we first present its PHY design that adopts directional beamforming to activate a specific sector, then its MAC frames that are used to set the duration field for silencing/activating nodes, and finally its MAC protocols for sector scheduling.

The basic idea of Wi-FiS is to divide sectors into timesharing access, i.e., only allowing nodes in a sector to contend channel each time. This method restricts the number of nodes for channel access and therefore reduces collision probability. The MAC layer protocol of Wi-FiS contains 3 stages: topology generation, sector generation, and sector scheduling.

With the help of Figure 5, we present the above three stages. (i) Topology generation: in this stage, the AP will periodically perform background scans to obtain information, i.e., direction angle and basic service set identifiers (BSSIDs), about surrounding nodes so as to calculate the physical location of each node and form a physical topology. To generate the entire topology, we need to obtain basic service set identifier (BSSID) of each node and its corresponding direction of arrival (DOA) through scans.

(1) Obtaining BSSID: in our design, the AP and nodes work in CSMA/CA and the AP acquires BSSIDs of nodes by scanning background flows in real time during the omnibeam uplink (UL) process. During the scanning, the AP will first extract BSSID field from the MAC header of a node's frame in the flows and then cache the BSSID to a database. However, a random BSSID mechanism affects obtaining a BSSID. To address the problem, we can automatically integrate a random BSSID with a real BSSID through a mechanism of matching RSSI (received signal strength indicator) and CSI (channel state information), reducing the impact of the random BSSID mechanism.

(2) Obtaining DOA: the AP can estimate DOAs using information extracted from physical headers of frames during the scanning of background flows, since nodes adopt multiantenna mechanism. This mechanism has been adopted by many wireless related technologies, e.g., [16-18]. When the AP receives a frame, it estimates DOA, associates DOA with BSSID extracted from the frame, and then caches them. Finally, the AP generates the physical topology according to DOA information and then divides the overall network into sectors according to the topology.

(3) Obtaining distance: during the association phase, the AP assigns the transmit power to all nodes, so the AP knows the transmit power of each packet. After receiving a packet, the AP obtains the receive power and further estimates the distance according to the transmit and receive power and the RSSI channel attenuation model.

(ii) Sector generation: in this stage, after obtaining the physical topology, the AP will divide the network into several sectors according to a preset restricted number of nodes (i.e., $n_{k}$ ) and the topology information. This sectorization method makes it possible for the AP to later schedule each sector via directional beamforming. Wi-FiS does not adopt equal partitioning method in hard disk. To ensure the network performance of each sector, we set the maximum number of nodes in each sector to $n_{k}$. Usually, we preset $n_{k}$ based on the actual network topology, packet size, and the theoretical performance model (mentioned in Section 6). As shown in 


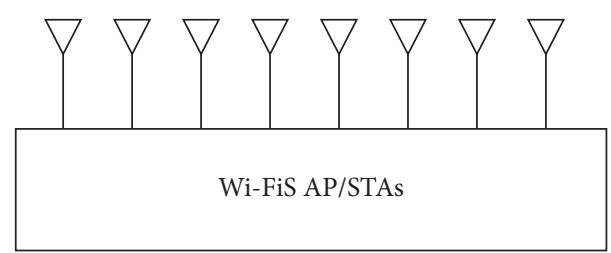

FIgURE 4: Hardware design of a AP/STA adopted in Wi-FiS.

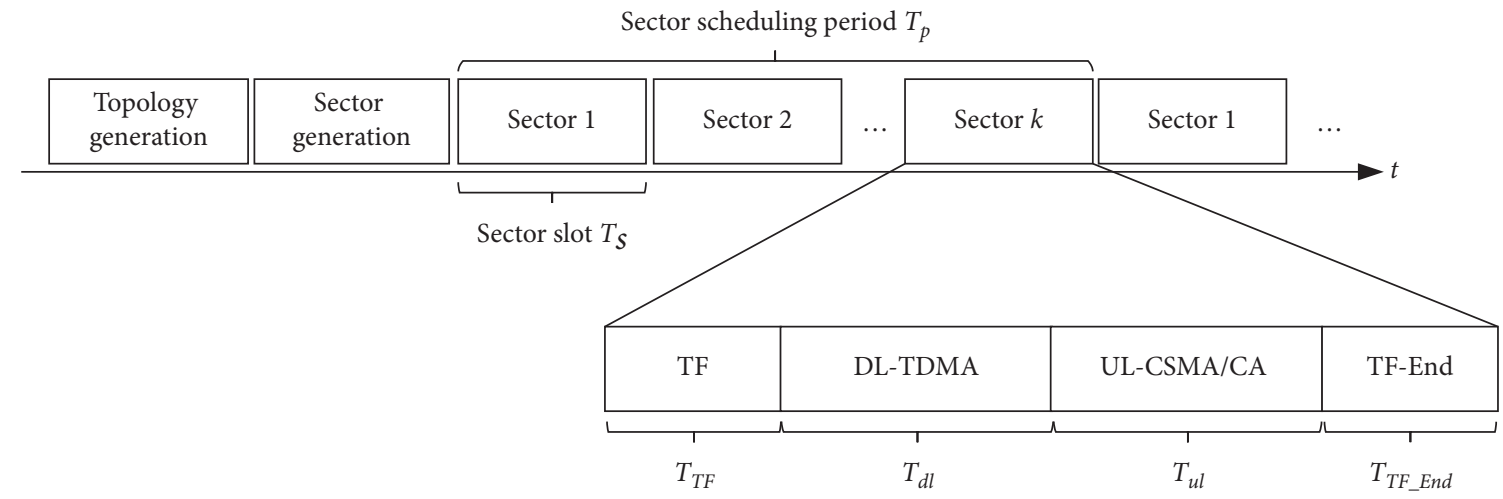

Figure 5: MAC protocol overview.

Figure 2, we obtain partitions via the control of arc and set the number of nodes in each sector to a fixed constant value. If the distribution of nodes in a sector is looser, the arc of the sector will be larger, and if the distribution of nodes is tighter, the arc is smaller.

Besides, our sector partition uses an incremental partition strategy, which can be divided into the following two steps.

(1) Step 1. Divide the total number of nodes $N$ by $n_{k}$ and then add 1 to the integer part of the result (i.e., dealing the result through a ceiling function). The integer part of result represents a saturated number of nodes in a sector, which is equal to $n_{k}$.

(2) Step 2. Let the remainder of $N$ divided by $n_{k}$ be the number of nodes for the last sector. Since the number of nodes in this sector is less than those of other sectors, the throughput of this sector will be slightly smaller. In our design, we adopt the same control mechanism for all sectors rather than introducing a specific control mechanism for this sector.

With the above strategy, the AP divides all nodes into sectors. After sectorization, they will enter the channel access stage.

(iii) Sector scheduling: in this stage, after the generation of sectors, the AP will schedule each sector. The AP determines whether there is an overlap between APs by listening to the TF frames of other APs. If yes, there will be no hidden/exposed terminals; otherwise, there possibly exists hidden/exposed terminals. Let $T_{p}$ be the scheduling time of all the sectors.
$T_{p}$ is a total time of $k$ sectors' operation time, where time for each sector (i.e., sector slot in Figure 5) is $T_{s}$. To schedule one sector, we make use of two control frames, i.e., trigger frame (TF) and TF-End frame. Note that we adopt omnibeam or sector beam for different frame types in this design to achieve scheduling for a specific area. Each sector slot process includes four processes, i.e., TF frame, downlink TDMA access (DL-TDMA), uplink CSMA/CA access (UL-CSMA/CA), and TF-End frame.

For a single-cell network, we may adopt omnibeam to transmit data during the DL-TDMA and ULCSMA/CA processes. In contrast, for a multicell network, we may adopt sector beam to transmit data during DL-TDMA and UL-CSMA/CA processes to avoid hidden and exposed terminal problems.

3.3. MAC Frame Settings. Conventional Wi-Fi has three types of Wi-Fi frames: management, control, and data frames. Here, we present their settings for silencing/activating nodes in $\mathrm{Wi}-\mathrm{FiS}$.

In the design of Wi-FiS, NAV parameters are set based on the duration/AID field. In order to implement the sectorbased time-sharing contention strategy, we need to control the duration/AID field of the frame sent by AP/STA. In 802.11 standard, the duration/AID field has two different functions. As shown in Figure 6, if the highest bit is set to 0, it is used as the duration filed, and the node will receive a frame including this field and update it to the node's own NAV timer; oppositely, if the highest bit is set to 1, then this field is used as an AID, and the node will not update this field to its NAV timer. 

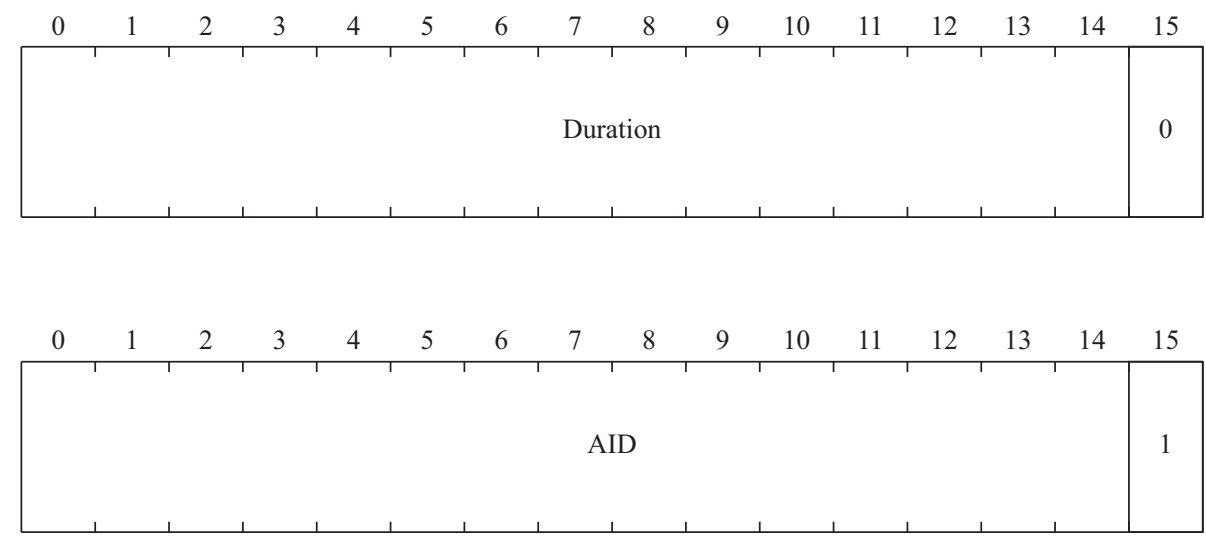

FIGURE 6: Duration/AID field in a 802.11 frame.

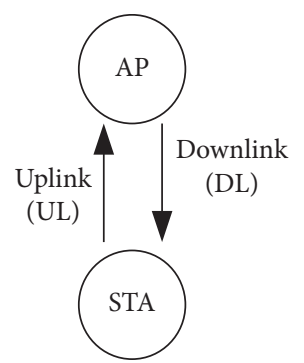

(a)

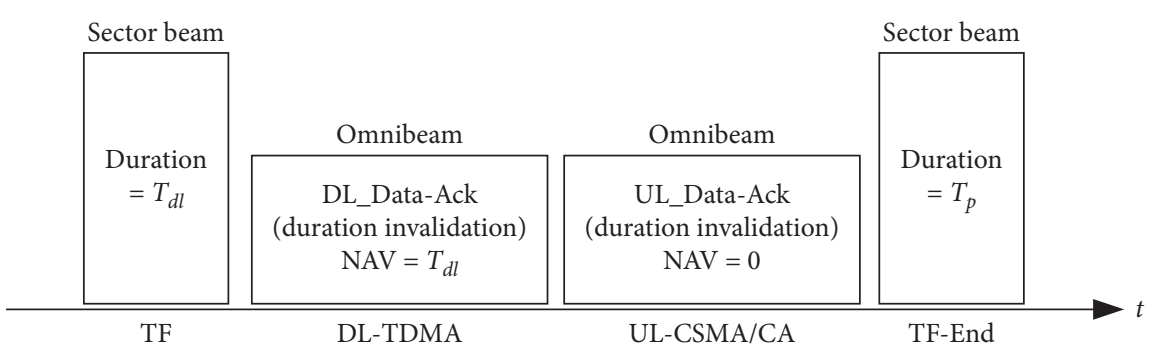

(b)

Figure 7: An overview of sector scheduling overview.

Therefore, in this design, due to the reason that we need to use APs for global scheduling, we use different types of duration/AID field to set mechanisms for frames of different objects.

(i) Frames sent by AP.

(1) TF and TF-End frames: the duration/AID field is set as the duration parameter for purpose of implementing partition scheduling.

(2) Data and control (ACK) frame: control frames include ACK and other different frames, and the data frames can be downlink data. Here, the AP sets the duration/AID field in these frames as the AID parameter. For example, the duration/AID field in both the ACK and downlink data frames can be set to the target AID. In the 802.11 protocol, if the duration/AID field is set to AID, then no AID analysis is performed.

(ii) Frames sent by STA: since nodes are scheduled objects, the duration/AID fields of the management frames (such as probes, association requests, and so on) sent by the node should be set to AID. A node may not have the corresponding AID value when it is not associated with an AP, and thus this value can be filled with 0 . The control frame and data frame sent by the node are the same as the control frame and data frame sent by the AP and should be set to the target AID.

\section{Wi-FiS Scheduling for Single Cell}

Here, we present the Wi-FiS scheduling method in a single cell.

4.1. Overview. In the 802.11 protocol, the AP may prohibit nodes from contending for the channel by setting nodes to the NAV state and then manipulate the entire transmission process. In the initial state, the NAV timer of all nodes is set to the maximum value. As mentioned in Section 3.2, the entire scheduling process of each sector includes four processes (as shown in Figure 7), trigger frame (TF) process, downlink TDMA (DL-TDMA) process, uplink CSMA/CA (UL-CSMA/CA) access, and TF-End process. The TF and TF-End processes are used to control NAV settings, while the DL-TDMA and UL-CSMA/CA data transmissions do not affect NAV. Below, we detail each process.

(i) TF process: at the beginning of each sector switching process, using a TF frame, the AP will schedule a sector $k$ to operate and set the corresponding competition and transmission parameters for nodes in this sector, where the transmission time of the TF frame is $T_{f}$. After receiving the TF frame, the nodes in the sector $k$ area will set their NAV to $T_{\mathrm{dl}}$, which means that the AP will take the next $T_{\mathrm{dl}}$ time for DL-TDMA data transmission.

(ii) DL-TDMA process: after the TF process, the AP will first perform the downlink data transmission. The 
downlink data are transmitted back-to-back in the order of TDMA and the nodes' AIDs, where the time of DL-TDMA is $T_{\mathrm{dl}}$. The NAV timer of the node in sector $k$ decreases with time, and when the DL-TDMA transmission process ends, this NAV timer is decreased to zero and therefore the nodes in sector $k$ can contend for the channel freely.

(iii) UL-CSMA/CA process: after the DL-TDMA process, nodes in the sector can perform uplink competition, which is based on the CSMA/CA protocol. After a node wins the competition, it can transmit uplink data to the AP, where the time of UL-CSMA/CA is $T_{\mathrm{ul}}$.

(iv) TF-End process: after the UL-CSMA/CA process, the AP will deactivate the sector. It will send a TFEnd frame to set all nodes in the sector to NAV state, and the sector cannot be activated again until the next sector scheduling period.

In our design, TF and TF-End frames are transmitted in the form of sector beam, which is used to schedule a specific sector. The data transmissions, that is, DL-TDMA and ULCSMA/CA processes, do not have the scheduling function. Therefore, omnibeam transmission can be performed. As described in Section 3.3, only the duration/AID fields for TF and TF-End frames are set to duration, while the data and ack packets in DL-TDMA and UL-CSMA/CA are set to AID. Therefore, only TF and TF-End affect the NAV settings, while data and ACKs do not.

In these two processes, the duration/AID fields of data and ACK frames are set for AID function, and hence NAV will not be affected. However, to avoid hidden/exposed terminal problems for multicell scenarios, we will change to sector-beam transmission for the data transmission process when these problems happen.

4.2. TF. At the beginning of each sector switching process, the AP will send TF frames via sector beam. Here, a TF frame contains the following two fields (as shown in Figure 8):

(i) Duration/AID field: it silences all nodes by setting them to NAV state.

(ii) Wi-FiS parameter element field: this field is used to notify and configure the transmission parameters of nodes in one sector, i.e., $\mathrm{CW}$, sector ID, $T_{s}$, and $T_{\mathrm{dl}}$.

(iii) CW: this field configures the contention parameter used by the node (similar to EDCA Parameter [19] of 802.11e). In our design, the AP can set the value of $\mathrm{CW}$ in the Wi-FiS parameter element field.

(iv) Sector ID: this field identifies the ID of the current scheduled sector by a TF frame and is used to let other APs know which sector is currently scheduled. Further, it determines whether there are hidden/ exposed terminal problems.

(v) $T_{s}$ (sector slot) and $T_{\mathrm{dl}}$ (DL-TDMA): these two fields identify the time of the entire sector scheduling time and the corresponding downlink TDMA scheduling time. They are used to allow other APs to determine whether there are hidden terminals or exposed terminal problems and the time when the problems occur, since $T_{\mathrm{ul}}$ can be obtained through $T_{s}-T_{\mathrm{dl}}-T_{\mathrm{TF}}-T_{\mathrm{TF} \_ \text {End }}$, where $T_{\mathrm{TF}}$ and $T_{\mathrm{TF} \_ \text {End }}$ are constant values.

Remark: usually CW is used in single-cell sector scheduling scenario, while sector ID, $T_{s}$, and $T_{\mathrm{dl}}$ are used in multicell sector scheduling scenario.

4.3. DL-TDMA. As soon as TF frame transmission ends, the downlink TDMA transmission starts and will last for $T_{d l}$ time. In our design, the downlink process adopts TDMA. The reasons are as follows. In downlink, the AP can allocate the access time slice of TDMA to nodes, and therefore there is no need for contention. Further, the AP can optimize the time slice allocation of TDMA according to the local cache information, and therefore TDMA is more efficient.

During this time period, the AP will sequentially send DL-DATA 1 to node 1 (i.e., AID = 1), DL-DATA 2 to node 2, and so on in the order of TDMA. The time interval between DL-DATA is equal to SIFS. The AP will transmit the downlink data of all nodes at one time, and during this process, the nodes will not actively feed back ACK. When the AP sends downlink data, it will record the sending sequence on the local bitmap.

After the downlink data transmission, the AP will broadcast an ACK request (ACK Req.) to request ACK feedback from all nodes. After the nodes receive the ACK Req., each of them will sequentially feed back an ACK to the AP according to corresponding AID sequence of the bitmap in the ACK Req. If the node successfully receives the downlink data, then it will feed back an ACK. If the node fails to receive the data, it needs to feed back an NACK (that is, the ACK indicates that the reception failed). When all nodes finish feeding back ACKs, the DL-TDMA process ends.

In our design, $T_{\mathrm{dl}}$ is set based on the current downlink traffic. In the DL-TDMA process, AP will first determine whether there is a downlink packet toward a node based on its local cache. If yes, the AP sends the packet to the node; otherwise, it checks if there is a downlink packet toward the next node. In this paper, we assume the downlink is saturated. So, the transfer time is equal to $n_{k}$ times the roundtime of a data-ack transmission. Therefore, it can set the time duration of this transmission, $T_{\mathrm{dl}}$.

4.4. UL-CSMA/CA. After the downlink transmission, the nodes in the current sector can perform uplink contention (i.e., UL-CSMA/CA process), and the time for UL-CSMA/ CA is $T_{\text {ul }}$. In our design, the uplink process adopts CSMA/ CA. The reasons are as follows. The uplink transmission should be based on contention, adapting to the change of traffic load, while CSMA/CA is a very successful contention protocol that is widely adopted in conventional 802.11 networks. Further, for uplink, CSMA/CA is more efficient than TDMA, since the AP does not know about the node's uplink traffic information and therefore cannot allocate 


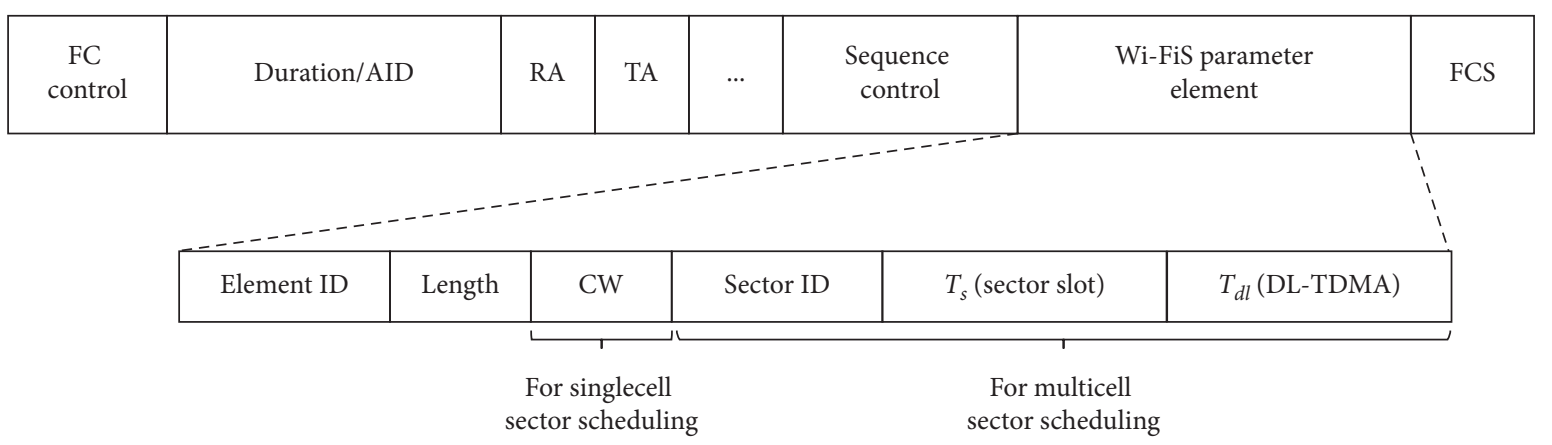

Figure 8: Structure of a trigger frame (TF).

access time to each node. As a result, it is better to let nodes contend for data transmission if they have packets. In this process, the NAV $=0$ of a node in the sector indicates that the node is activated and can compete for channel freely. $\mathrm{NAV}=T_{p}$ of the node in other sectors indicates that the node is in the silence state.

The nodes in the sector compete for channel according to the standard backoff process. Each node will first select a random value range from $[0, \mathrm{CW}]$ for its Backoff cOunter (BO). The selected value is stored in the BO. Every time there is an idle slot passing, $\mathrm{BO}$ will count down one once. When a node's BO counts down to 0 , it means the node wins the channel contention. Hence, the node can send its uplink data to the AP. When the AP receives the data and verifies that it is correct, it will feed back an ACK to the node; otherwise, if the node fails to receive the data, it needs to feed back a NACK [20].

In the UL-CSMA/CA process, the random access-based CSMA/CA mechanism makes it unable to determine the end of this process. However, to set fixed time duration of the UL-CSMA/CA process, $T_{\text {ul }}$, in our design, we will keep a holding time duration, $T_{h}$, for each end of a UL-CSMA/CA process. Within this time $\left(T_{h}\right)$ range, all nodes will be set to the silence state, and they will not perform backoff and data transmission. The setting of $T_{h}$ refers to the necessary time required for one minimum complete transmission (including the average backoff time and the time of data transmission and ACK). In order to realize the protection mechanism of the holding time, we need the following settings based on the ACK mechanism.

(i) If $T_{r} \geq T_{h}$, the AP can only feed back ACK, which means that the remaining time of the UL-CSMA/CA process is enough for one complete transmission.

(ii) If $T_{r}<T_{h}$, after the AP feeds back the ACK, it will send padding frames to occupy the channel, so that the next round of transmission cannot start for the remaining time (because the remaining time is not enough to guarantee one complete transmission). The duration of the padding frame transmission is $T_{r}$. Since the channel is always occupied by the AP, all nodes in the sector will not be able to perform backoff until the next UL-CSMA/CA process of this sector.
Unlike downlink transmission, the AP cannot accurately get the real-time buffering situation of all nodes in uplink access. Therefore, the AP sets uplink UL-CSMA/CA time duration $T_{\mathrm{ul}}$ to a fixed value regardless of nodes' buffering situation.

4.5. TF-End. At the end of each sector switching process, the AP will use sector beam to send TF-End frames. In the TFEnd frame, the AP will set the duration to $T_{p}$ (as shown in Figure 9).

\section{Wi-FiS Scheduling for Multicell}

In the multicell scenario of the wireless network, there are two basic problems: (1) hidden terminal problem and (2) exposed terminal problem. In this section, we take a two-cell topology as an example for discussion, and each cell contains $1 \mathrm{AP}$ and 1 node (namely, $\mathrm{AP}_{1}$ and $\mathrm{N}_{1}$ and $\mathrm{AP}_{2}$ and $\mathrm{N}_{2}$, respectively).

5.1. Overview. Here, we outline Wi-FiS scheduling method in multicell. In the multicell scenario, the AP of the cell (such as $\mathrm{AP}_{1}$ ) will actively receive $\mathrm{TF}$ frames from APs of other cells (such as $\mathrm{AP}_{2}$ ). The AP determines if there is an overlap between the two cells by receiving the TF frames of other APs. If yes, there might exist hidden/exposed terminal problems; otherwise, there are no such problems. In order to solve the problem of hidden/exposed terminals on $\mathrm{AP}_{x}$, we need to dynamically adjust the $\mathrm{AP}_{\mathrm{x}}$ to perform sector-beam transmission rather than omnidirectional transmission in a single cell (as shown in Figure 10). To achieve this, we need to determine whether the hidden/exposed terminal will happen and how long it will last for. Therefore, we need to know the relative position of the APs in other cells and how long their relative scheduling time is.

(i) Obtaining relative position of APs in other cells: we can get relative position of APs through DOA and distance measurement-related technologies [16-18]. Further, based on this position information, we can determine whether there will be hidden/exposed terminal problems between $\mathrm{AP}_{1}$ and $\mathrm{AP}_{2}$. 


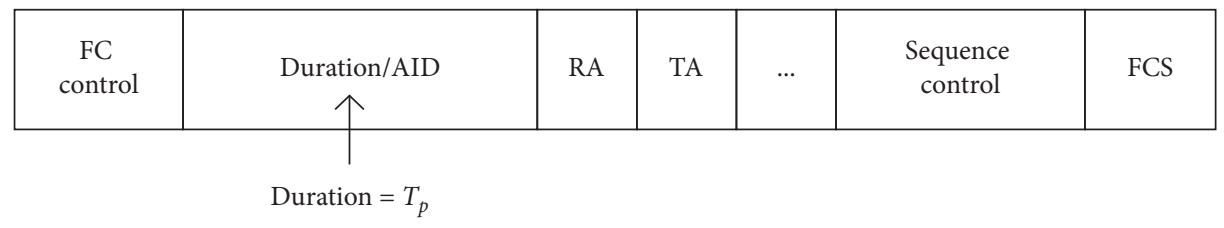

FIgURE 9: The structure of a TF-End frame.

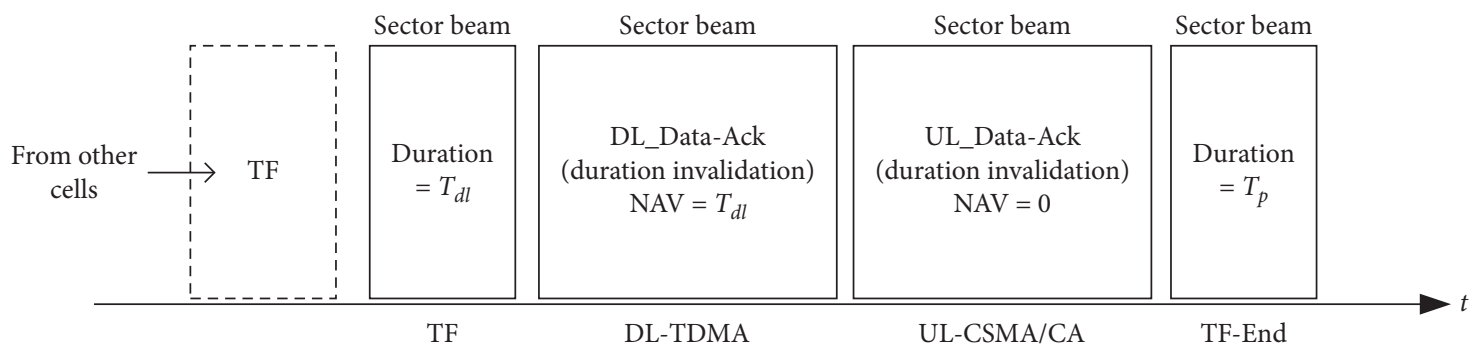

Figure 10: Wi-FiS scheduling for multicell scenario.

(ii) Obtaining sector scheduling time of other cells: since we include the sector ID, the total transmission time $T_{s}$ of the sector, and the DL-TDMA time $T_{\mathrm{dl}}$ in the TF frame, we can further deduce the time duration of hidden/exposed terminals.

Based on the above information, in the sector scheduling stage, in addition to the time scheduling relative to single cell (such as $T_{\mathrm{dl}}$ and $T_{\mathrm{ul}}$ time), to avoid hidden terminal and exposed terminal problems, we can use sector beam for data transmission. However, it should be noted that the sector-beam method requires extra overhead for a certain measurement (such as CSI measuring) to operate precoding. Therefore, in a single-cell scenario, or in a scenario where there is no hidden/exposed terminal, we can directly adopt omnibeam transmission. Below, we, respectively, describe the problem of hidden/exposed terminal and its solution.

\subsection{Hidden Terminal Problem and Solution}

5.2.1. Hidden Terminal Problem. Taking Figure 11 as an example, since $\mathrm{AP}_{1}$ is in the coverage of both $N_{1}$ and $\mathrm{AP}_{2}$, when $N_{1}$ uses an omnidirectional antenna to transmit uplink data to $\mathrm{AP}_{1}$ and $\mathrm{AP}_{2}$ uses an omnidirectional antenna to transmit downlink data to $N_{2}, \mathrm{AP}_{1}$ will receive the superimposed signals of $N_{1}$ and $\mathrm{AP}_{2}$ at the same time. Therefore, the signal cannot be demodulated, which causes a collision. Such collision problem is called as hidden terminal problem.

Solution. $\backslash s \backslash$ up2 ( $1(1) ?^{1}$ : at the beginning of the network operation, $\mathrm{AP}_{1}$ will first broadcast a $\mathrm{TF}$ (denoted by $\backslash s \backslash$ up2(1(1)? ${ }^{1}$ in the figure), which announces that the network covered by $\mathrm{AP}_{1}$ will perform uplink transmission.

$\backslash s \backslash$ up2( $2 a(2 a) ?^{2 a}$ : after $\mathrm{AP}_{2}$ receives the TF, it will use directional beam transmission when performing downlink transmission, that is, beam-downlink (denoted by $\backslash s \backslash$ up2( $2 a(2 a) ?^{2 a}$ in the figure above).

$\backslash s \backslash$ up2( $2 b(2 b) ?^{2 b}: \mathrm{AP}_{1}$ will also allow the local node to perform uplink transmission after transmitting the TF. As shown in the figure, $N_{1}$ uses broadcast to perform uplink transmission (denoted by $\backslash s \backslash \operatorname{up} 2\left(2 b(2 b) ?^{2 b}\right.$ in the figure).

\subsection{Exposed Terminal Problem and Solution}

5.3.1. Exposed Terminal Problem. Taking Figure 12 as an example, the network covered by $\mathrm{AP}_{2}$ includes both $\mathrm{AP}_{1}$ and $N_{2}$. When $\mathrm{AP}_{1}$ uses an omnidirectional antenna for downlink transmission, $\mathrm{AP}_{2}$ will actively receive the transmitted signal by $\mathrm{AP}_{1}$. The signal can be either one of the following two frames: (1) a trigger frame (TF) that $\mathrm{AP}_{2}$ needs to receive; (2) a data frame that $\mathrm{AP}_{2}$ does not need to receive. Then, if the signal is a data frame from $\mathrm{AP}_{1}$, the receiving of the signal causes a waste of channel utilization for $\mathrm{AP}_{2}$ because the channel used for receiving these data can be used for $\mathrm{N}_{2}$ 's downlink transmission instead. Such a waste problem is called an exposed terminal problem.

Solution. $\backslash s \backslash$ up2 ( $1(1) ?^{1}$ : at the beginning of the network operation, $\mathrm{AP}_{1}$ will first broadcast a $\mathrm{TF}$ (denoted by $\backslash s \backslash$ up2(1(1)? ${ }^{1}$ in the figure), which announces that the network covered by $\mathrm{AP}_{1}$ will perform directional downlink transmission.

$\backslash s \backslash$ up2 $2 a(2 a) ?^{2 a}$ : after $\mathrm{AP}_{2}$ receives the $\mathrm{TF}$, it learns that the $\mathrm{AP}_{1}$ will perform directional downlink transmission, and the node that is transmitting data is not within the coverage of $\mathrm{AP}_{2}$. At this point, $\mathrm{AP}_{2}$ can perform downlink transmission in form of broadcasting (denoted by $\backslash s \backslash$ up2( $2 a(2 a) ?^{2 a}$ in the figure above). 
$\backslash s \backslash$ up2 $\left(2 b(2 b) ?^{2 b}\right.$ : after $\mathrm{AP}_{1}$ finishes transmitting the TF frame, it will directly use a directional beam to perform downlink transmission (denoted by $\backslash s \backslash$ up2 $\left(2 b(2 b) ?^{2 b}\right.$ in the figure) and transmit data to node $\mathrm{N}_{1}$ directionally.

\section{Theoretical Analysis}

\subsection{Th(K): Total Throughput}

Assumption 1. We assume that an AP passively collects topology information, so the topology generation process does not need to spend additional channel time. And because the sector generation process is done locally, it does not need to occupy the channel resource.

Assumption 2. Both the uplink and downlink transmissions of the system are saturated.

In this section, we analyze the performance of the proposed Wi-FiS protocol. Under Assumptions 1 and 2, we define the system throughput as follows:

$$
T h(K)=\frac{\sum_{k=1}^{K}\left(T h_{k}\left(n_{k}\right) \cdot T_{s}\right)}{T_{p}},
$$

where $k$ represents the $k$-th sector $k=1,2,3, \ldots, K), T_{p}$ is the time of a sector scheduling period, $T_{s}$ is the scheduling time of a sector, and $T h_{k}\left(n_{k}\right)$ represents the relative throughput of the $k$-th sector.

6.2. $\mathbf{T h}_{\mathbf{k}}\left(\mathbf{n}_{\mathbf{k}}\right)$ : Throughput of Sector $\mathbf{k}$. Since our design includes downlink transmission (i.e., DL-TDMA) and uplink contention transmission (i.e., UL-CSMA/CA), we need to calculate the throughput of these two parts separately.

Assumption 3. The fixed length of the data frame is $L_{\mathrm{MPDU}}$. Let $T h_{-} \mathrm{DL}_{k}\left(n_{k}\right)$ denote the downlink throughput of DLTDMA and let $T h_{-} \mathrm{UL}_{k}\left(n_{k}\right)$ denote the uplink throughput of UL-CSMA/CA. Therefore, under Assumption 3, the relative throughput of sector $k$ can be calculated as

$$
T h_{k}\left(n_{k}\right)=\frac{T h_{\mathrm{DL} k}\left(n_{k}\right) \cdot T_{\mathrm{dl}}+T h_{\mathrm{UL} k}\left(n_{k}\right) \cdot T_{\mathrm{ul}}}{T_{s}} .
$$

6.3. $\mathbf{T h}_{-} \mathbf{D L}_{\mathbf{k}}\left(\mathbf{n}_{\mathbf{k}}\right)$ : Downlink (DL-TDMA) Throughput of Sector k. In this section, we describe the downlink (i.e., DLTDMA) throughput calculation of sector $k$. Referring to Figure 13, the entire downlink transmission time $T_{\mathrm{dl}}$ depends on the maximum number of transmissions $M$ in this period.

Let $M$ denote the total number of downlink transmissions in this sector, and the value of $M$ can be calculated as follows:

$$
M=\frac{T_{\mathrm{dl}}-\left(L_{\mathrm{ACK}-\text { Req. }} / R+t T_{\mathrm{SIFS}}\right)}{2 * T_{\text {PHY_Header }}+\left(L_{\mathrm{MPDU}}+L_{\mathrm{Ack}}\right) / R+T_{\text {SIFS }}},
$$

where $T_{\mathrm{dl}}$ is the total time of downlink transmission, $R$ is the data transmission rate, $T_{\mathrm{PHY}} \mathrm{H}_{\text {Header }}$ is the transmission time of the physical layer header, $L_{\mathrm{MPDU}}$ is the size of the entire payload, $L_{\text {ACK-Req. }}$ is the size of the ACK request frame, $L_{\text {Ack }}$ is the size of the ACK frame, and $T_{\text {SIFS }}$ is the size of the SIFS time interval.

Assumption 4. The total downlink transmission time $T_{\mathrm{dl}}$ is set depending on the number of transmissions $M$.

In our design, the total number of downlink transmissions $M$ is set to the number of nodes in a sector, $n_{k}$, that is, $M=n_{k}$. So, under Assumption 4, the throughput of downlink transmission is as follows:

$$
T h \_L_{k}\left(n_{k}\right)=\frac{L_{\mathrm{MPDU}} * n_{k}}{T_{\mathrm{dl}}} \text {. }
$$

6.4. $\mathbf{T h}_{-} \mathbf{U L}_{\mathbf{k}}\left(\mathbf{n}_{\mathbf{k}}\right)$ : Uplink (UL-CSMA/CA) Throughput of Sectork. In this section, we describe the uplink (UL-CSMA/ CA) throughput calculation of sector $k$. Referring to Figure 14, the CSMA/CA contention mode is adopted in the uplink process. We first calculate the total number of successful uplink transmissions $M$ with the following formula:

$$
M=E_{M_{\mathrm{tr}} \mid N}\left(n_{k}\right) \cdot P_{\mathrm{SUC} \mid N}\left(1 \mid n_{k}\right), \quad \text { when }\left(n_{k} \geq 2\right),
$$

where $E_{M_{\mathrm{tr}} \mid N}\left(n_{k}\right)$ represents the average number of attempts to transmit data packets in sector $k$ (under the condition of $\left.N=n_{k}\right)$ and $P_{\mathrm{SUC} \mid N}\left(1 \mid n_{k}\right)$ represents successful transmission in sector $k$ (under the condition of $N=n_{k}$ ). Thus, we have:

$$
\begin{aligned}
\operatorname{Th}_{-} \mathrm{UL}_{k}\left(n_{k}\right) & =\frac{L_{\mathrm{MPDU}} * M}{T_{\mathrm{ul}}} \\
& =\frac{L_{\mathrm{MPDU}} * E_{M_{\mathrm{tr}} \mid N}\left(n_{k}\right) \cdot P_{\mathrm{SUC} \mid N}\left(1 \mid n_{k}\right)}{T_{\mathrm{ul}}} .
\end{aligned}
$$

Below we need to calculate $E_{M_{\mathrm{tr}} \mid N}\left(n_{k}\right)$ and $P_{\mathrm{SUC} \mid N}\left(1 \mid n_{k}\right)$.

$$
\mathbf{P}_{\text {SUC } \mid \mathbf{N}}\left(1 \mid \mathbf{n}_{\mathbf{k}}\right) \text {. }
$$

Based on the standard Bianchi model, we can calculate the collision probability $p$ and the transmission probability $\tau$ :

$$
\begin{aligned}
& p=1-(1-\tau)^{n_{k}-1}, \\
& \tau=\frac{E[R]}{E[B]+E[R]},
\end{aligned}
$$

where $E[R]$ and $E[B]$ can be calculated as follows: 


$$
\begin{aligned}
E[R]= & \sum_{r=1}^{R_{\max }-1} r(1-p) p^{r-1}+R_{\max } p^{R_{\max }-1}=\sum_{r=1}^{R_{\max }} p^{r-1} \\
E[B]= & \frac{1}{2} \sum_{r=1}^{R_{\max }-1} \min \left\{2^{r-1} \mathrm{CW}_{\min }, \mathrm{CW}_{\max }\right\}(1-p) p^{r-1} \\
& +\frac{1}{2} \min \left\{2^{R_{\max }-1} \mathrm{CW}_{\min }, \mathrm{CW}_{\max }\right\} p^{R_{\max }-1} \\
= & \frac{1}{2} \sum_{r=1}^{R_{\max }} \min \left\{2^{r-1} \mathrm{CW}_{\min }, \mathrm{CW}_{\max }\right\} p^{r-1} .
\end{aligned}
$$

In the above formulas, $R_{\max }$ is the maximum number of transmission attempts. Under normal circumstances, we can set it to 5, which means that after the seventh transmission failure of a node, the packet loss will occur directly.

By solving $p$ and $\tau$ simultaneously using the above equations, we can calculate the probability of successful transmission $P_{\mathrm{SUC} \mid N}\left(1 \mid n_{k}\right)$, namely,

$$
\begin{gathered}
P_{\mathrm{SUC} \mid N}\left(1 \mid n_{k}\right)=\frac{n_{k} \tau(1-\tau)^{n_{k}-1}}{1-(1-\tau)^{n_{k}}}, \\
\mathbf{E}_{\mathbf{M}_{\mathbf{t r}} \mid \mathbf{N}}\left(\mathbf{n}_{\mathbf{k}}\right),
\end{gathered}
$$

where $\mathbf{E}_{\mathbf{M} \mid \mathbf{N}}\left(\mathbf{n}_{\mathbf{k}}\right)$ can be calculated as follows:

$$
E_{M_{\mathrm{tr}} \mid N}\left(n_{k}\right)=\sum_{m_{\mathrm{tr}}=1}^{M_{\mathrm{tr}-\mathrm{upper}}\left(T_{\mathrm{ul}}-T_{h}\right)} m_{\mathrm{tr}} P_{M_{\mathrm{tr}} \mid N}\left(m_{\mathrm{tr}} \mid n_{k}\right),
$$

where $M_{\text {tr-upper }}$ is the upper bound of the number of transmissions in UL-CSMA/CA process, $T_{\mathrm{ul}}$ is the total uplink access transmission time, and $T_{h}$ is a fixed guard time overhead (as shown in Figure 14).

Then, we calculate $P_{M_{\mathrm{tr}} \mid N}\left(m_{\mathrm{tr}} \mid n_{k}\right)$ :

(i) When $1 \leq M_{\text {tr }} \leq M_{\text {tr-upper }}\left(T_{\mathrm{ul}}-T_{h}\right)$,

$$
\begin{aligned}
P_{M_{t r} \mid N}\left(m_{t r} \mid n_{k}\right)= & \operatorname{Prob}\left(M_{t r} \geq m_{t r}\right) \\
& -\operatorname{Prob}\left(M_{t r} \geq m_{t r}+1\right) .
\end{aligned}
$$

(ii) When $M_{\mathrm{tr}}=M_{\text {tr-upper }}\left(T_{\mathrm{ul}}-T_{h}\right)$,

$$
P_{M_{\mathrm{tr}} \mid N}\left(m_{\mathrm{tr}} \mid n_{k}\right)=\operatorname{Prob}\left[M_{\mathrm{tr}} \geq M_{\mathrm{tr}-\mathrm{upper}}\left(T_{\mathrm{ul}}-T_{h}\right)\right]
$$

where

$$
M_{\text {tr-upper }}\left(T_{\mathrm{ul}}-T_{h}\right)=\frac{T_{\mathrm{ul}}-T_{h}}{2 * T_{\mathrm{PHY} \_ \text {Header }}+\left(L_{\mathrm{MPDU}}+L_{\text {Ack }}\right) / R+T_{\text {SIFS }}},
$$

where $R$ is the data transmission rate, $T_{\text {PHY_Header }}$ is the transmission time of the physical layer header, $L_{\mathrm{MPDU}}$ is the size of the entire payload, $L_{\mathrm{ACK}-\text { Req. }}$ is the size of the ACK request frame, $L_{\text {Ack }}$ is the size of the ACK frame, and $T_{\text {SIFS }}$ is the SIFS time interval.

Assumption 5. The smallest unit on the entire timeline is the Wi-FiS slot.

To calculate $\operatorname{Prob}\left(M_{\mathrm{tr}} \geq m_{\mathrm{tr}}\right)$, under Assumption 5, we have

$$
\operatorname{Prob}\left(M_{\mathrm{tr}} \geq m_{\mathrm{tr}}\right)=\operatorname{Prob}\left\{T_{b, m_{\mathrm{tr}}}+T_{t, m_{\mathrm{tr}}} \leq T_{\mathrm{ul}}-T_{h}\right\} .
$$

According to [21], we stipulate that $T_{b, m_{\mathrm{tr}}}$ is the total backoff time during $m_{\mathrm{tr}}$ transmission (based on Assumption 4 , which is the total number of slots), $T_{t, m_{\mathrm{tr}}}$ is the total transmission time in the $m_{\mathrm{tr}}$ transmission process (including the total time of DIFS $d$, data packet, SIFS, ACK), and $\varphi$ is the time of one packet transmission process. Hence, we have the following.

$\operatorname{Prob}\left(M_{\mathrm{tr}} \geq m_{\mathrm{tr}}\right)=\sum_{z=m_{\mathrm{tr}}}^{T_{\mathrm{ul}}-T_{h}} \operatorname{Prob}\left\{\sum_{j=1}^{m_{\mathrm{tr}}} T_{b, j}=z \mid n_{k}\right\} . \quad$ Let $q^{\prime}=1-(1-\tau)^{g}$, so this formula can be further simplified as

$$
\begin{aligned}
\operatorname{Prob}\left(M_{\mathrm{tr}} \geq m_{\mathrm{tr}}\right) & =\operatorname{Prob}\left(T_{t, m-1}+d+T_{b, m}+1 \leq T_{\mathrm{ul}}-T_{h}\right) \\
& =\sum_{z=m_{\mathrm{tr}}}^{T_{u l}-T_{h}-(m-1)(\varphi+d)-d+1} \operatorname{Prob}\left\{\sum_{j=1}^{m_{\mathrm{tr}}} T_{b, j}=z \mid n_{k}\right\} \\
& =\sum_{k=0}^{T_{\mathrm{ul}}-T_{h}-(m-1) \varphi-d-1-m}\left(\begin{array}{c}
k+m-1 \\
m-1
\end{array}\right) q^{\prime m}\left(1-q^{\prime}\right)^{k} .
\end{aligned}
$$




\section{Performance Evaluation}

In this section, we evalute the performance of Wi-FiS.

7.1. Simulation Setting. We write $\mathrm{C}++$ codes to simulate the Wi-FiS protocol, and each simulation lasts for $100 \mathrm{~s}$. We assume that there are at most 100 nodes in the network covered by an AP. All nodes are partitioned according to a fixed number of nodes (i.e., $n_{k}$ ). As the number of nodes continues to increase, the number of partitions should increase accordingly. Since we are mainly concerned with the uplink traffic of the node, we assume the AP has no downlink traffic in Figures 15-20. The simulation parameters are shown in Table 1.

7.2. Single-Cell Network. Figure 20 plots the system throughput via the total number of nodes. Its $x$-axis denotes the total number of nodes, ranging from 1 to 100 , and its $y$ axis denotes the throughput in Mbps. In the figure, the blue line is the throughput in CSMA/CA, the red dotted line is the throughput in RTS/CTS, and the purple and yellow lines denote the throughput corresponding to the proposed $\mathrm{Wi}$ FiS in this article.

From a holistic perspective, we can see that the throughput of Wi-FiS is significantly better than that of CSMA/CA and RTS/CTS. From this figure, we have the following observations.

(i) As the number of nodes increases (within 5 nodes), the throughput of these four methods increases as the number of nodes increases. This is because the increase in the number of nodes will lead to the increase in the transmission probability of the node, and in the meantime, the collision probability still maintains a small value. So, it will not have a major impact on the throughput.

(ii) When the number of nodes is between 5 and 100, the throughput exhibits different trends for different schemes.

(1) The throughput of CSMA/CA drops sharply as the number of nodes increases. This is because the collision probability increases with the number of nodes. In the meantime, since collision overhead is the transmission time of the entire data frame, high overall collision overhead leads to throughput degradation. As the number of nodes increases, the throughput will continue to decline.

(2) The throughput of RTS/CTS decreases slowly as the number of nodes increases. Compared with the CSMA/CA mode, although the collision probability is also increased, the collision overhead in the RTS/CTS is less than the transmission time of the data frame. Therefore, RTS/CTS slows down the throughput degradation. We can see that the throughput of RTS/CTS is better than that of CSMA/CA for about 35 nodes.
(3) The throughput of Wi-FiS (downlink enabled) increases as the number of nodes increases. The reason is that the throughput of Wi-FiS (downlink enabled) is the weighted sum of uplink throughput and downlink throughput. Since there are no collisions in the downlink transmission, the throughput of it increases with the number of nodes, which in turn leads to an upward trend in the overall throughput of WiFiS.

(4) The throughput of Wi-FiS (downlink disabled) stays almost unchanged as the number of nodes increases (in fact, it decreases slowly). This is because we control the network through sector partition scheduling so that collisions among large-scale nodes contentions caused by simultaneous contention can be avoided. We set up to 5 nodes for each sector. Compared with the CSMA/CA, since we have reduced the number of nodes which perform contention for each unit of sector time (i.e., $T_{s}$ ), the collision probability is controlled within a limited range to ensure high throughput. Compared with the RTS/CTS mode, our transmission management overhead is for each unit of sector time (i.e., $T_{s}$ ), which requires 2 management frames (i.e., TF and action frames) and 2 SIFS, which is relatively smaller than RTS/CTS (each transmission requires a handshake). Therefore, the throughput will be higher than that in the RTS/CTS mode.

In the figure, we can also see that with about 5 nodes (namely, $n_{k}$ ), the Wi-FiS throughput will suddenly drop once and then gradually rise again. This is because we adopt an incremental partitioning strategy. For example, when there are 11 nodes, we will divide all nodes into 3 sectors, which are 2 sectors with 5 fixed nodes and a sector with 1 node. In the channel access process, time-sharing access is performed in accordance with 3 sectors, which will result in a decrease in the throughput of the sector with only one node, and finally weighted to a decrease in the overall throughput. However, as the number of nodes in this area increases, its throughput gradually rises. When the number of nodes continues to increase, the throughput will periodically drop due to the newly added sectors.

Figure 15 plots the theoretical and simulation throughput of the UL-CSMA/CA process as the number of total nodes varies from 1 to 100 , where the number of nodes in each sector, $n_{k}$, is 5 and 20 , respectively. The red solid line represents the theoretical throughput while the black dashed line is for the simulation throughput. From this figure, we have the following observations.

(1) The overall throughput when $n_{k}=5$ is higher than the overall throughput when $n_{k}=20$. The reason is that when the number of nodes in each 


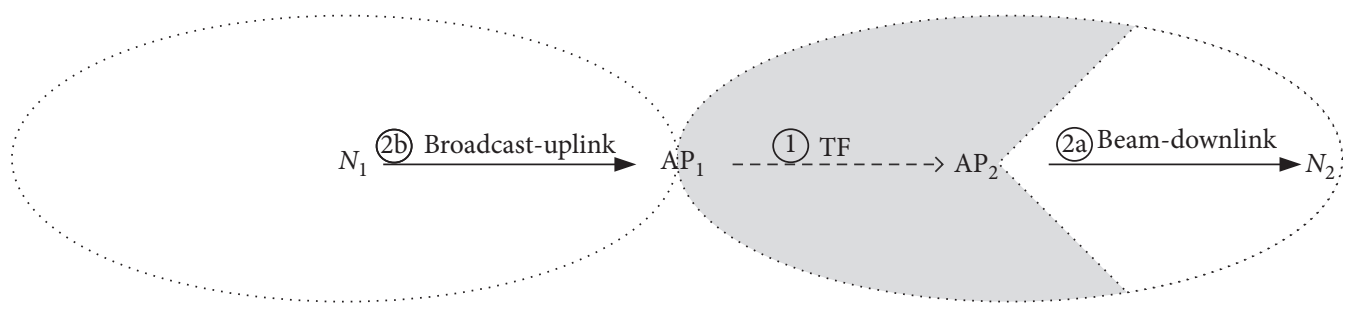

Figure 11: An example of hidden terminal problem and solution.

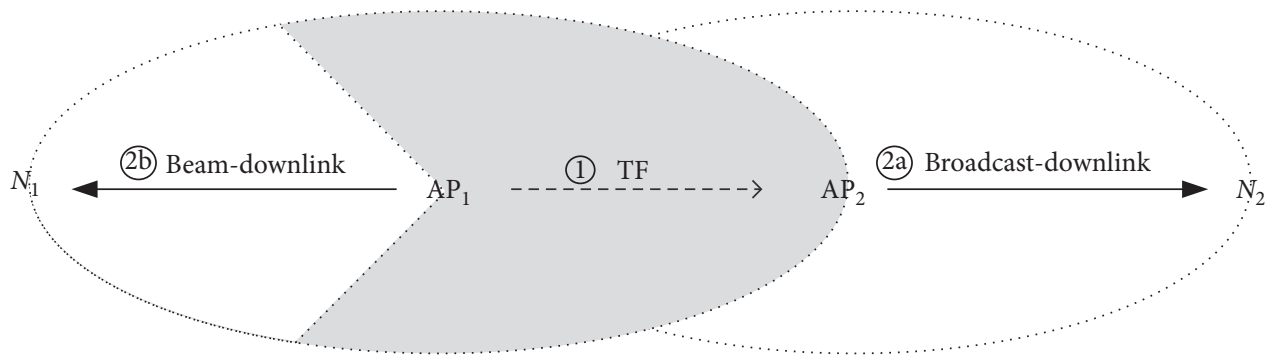

Figure 12: An example of exposed terminal problem and solution.

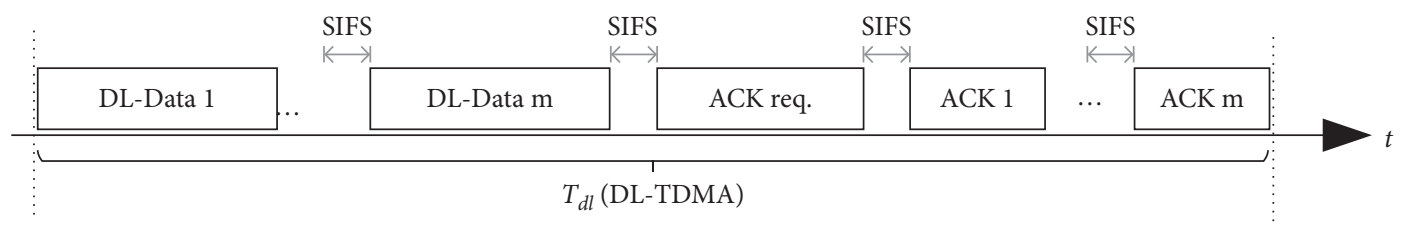

Figure 13: The downlink TDMA transmission (DL-TDMA) process.

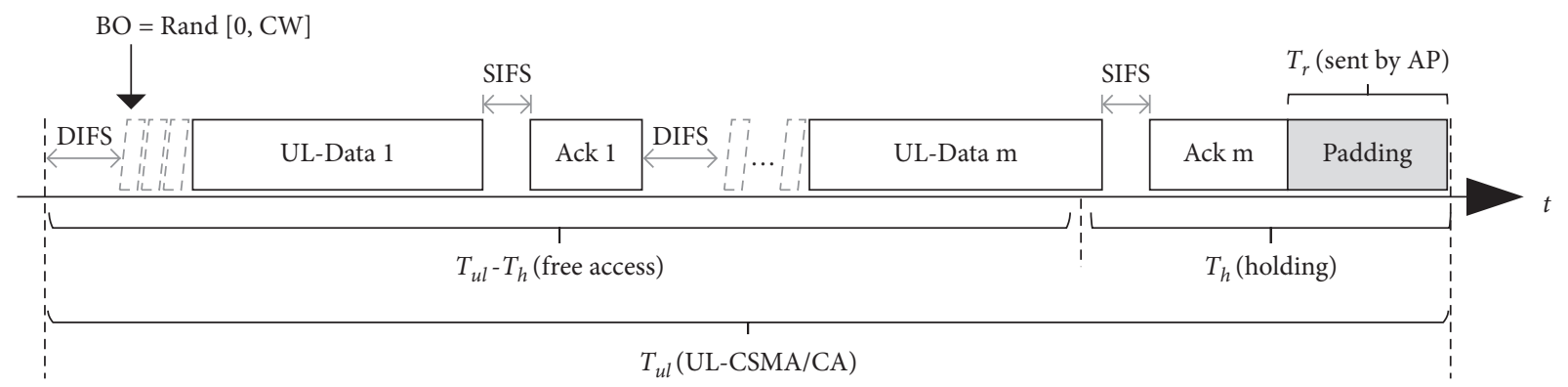

Figure 14: The uplink CSMA/CA (UL-CSMA/CA) transmission process.

sector is bigger, the collision probability for each sector will be higher, which decreases the throughput.

(2) For $n_{k}=20$, periodically the throughput first increases rapidly, then decreases slowly, and drops suddenly. The reason can be explained as follows. The throughput increases since the number of nodes increases and then decreases because the collision probability increases with node number increasing, which decreases the throughput. Following the same deduction, the reason for sudden throughput drop can be explained for $n_{k}=5$.

Figure 16 shows the throughput of the ULCSMA/CA process as the number of nodes varies from 1 to 100 , where the holding time $T_{h}$ is $500 \mu \mathrm{s}$ and $2000 \mu$ s, respectively. It can be observed from this figure that the overall throughput when $T_{h}=$ 


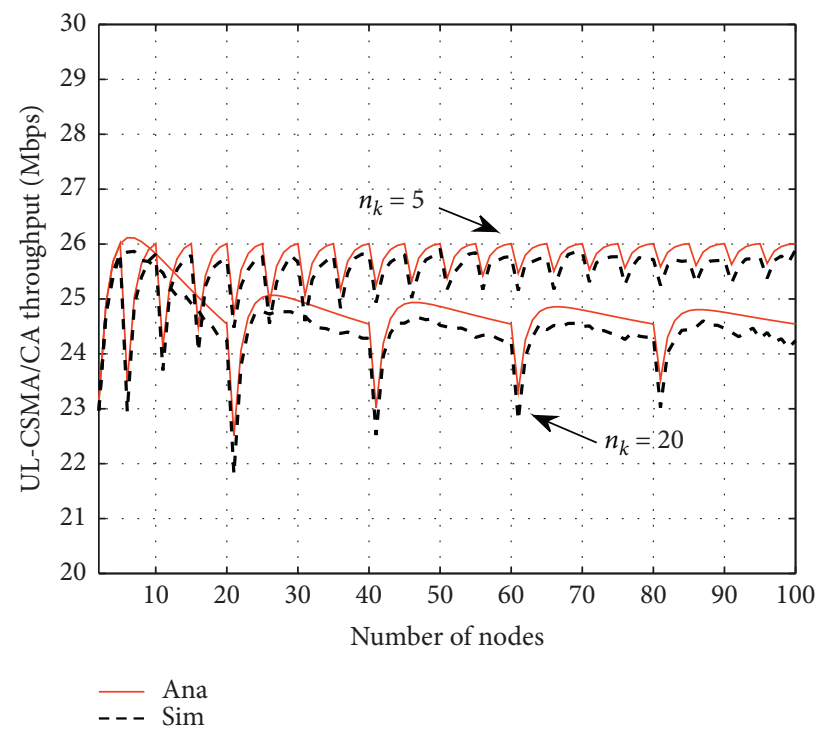

FIgURE 15: UL-CSMA/CA throughput vs. number of nodes, $N$, when $n_{k}=5,20$.

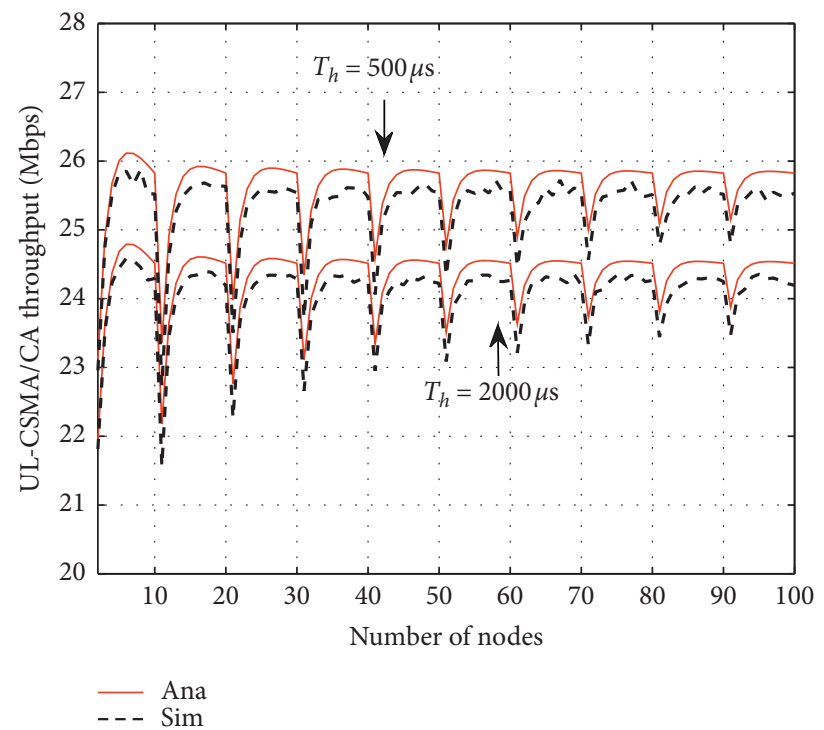

Figure 16: UL-CSMA/CA throughput vs. number of nodes, $N$, when $T_{h}=500$ and $T_{h}=2000$.

500 is higher than that when $T_{h}=2000$. It is because the longer the holding time is, the more the time that can be used for transmission is wasted. Besides, following the same deduction of Figure 15, we can explain the sudden drop in the throughput.

Figure 17 plots the throughput of the UL-CSMA/CA process as the number of nodes varies from 1 to 100 , where the uplink transmission time $T_{\mathrm{ul}}$ is set to $10000 \mu \mathrm{s}$ and $30000 \mu \mathrm{s}$, respectively. In this simulation, we set the holding time $T_{h}$ to a fixed value of
$500 \mu \mathrm{s}$. It can be seen from this figure that the throughput when $T_{\mathrm{ul}}=30000$ is higher than that when $T_{\mathrm{ul}}=10000$. The reason is that the longer the time for the uplink transmission is, the more the data transmission there is and therefore the higher the throughput is.

Figure 18 plots the throughput of the UL-CSMA/CA process as the payload length varies from 200 to 2000 bytes, where the number of each sector, $n_{k}$, is 5 and 20 , respectively. From this figure, we have the following observations. 


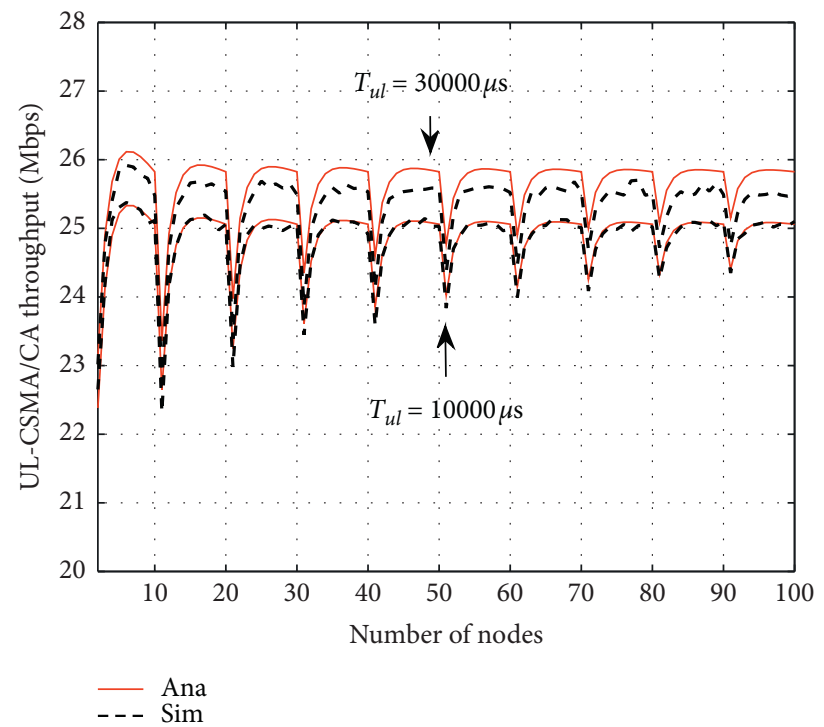

Figure 17: UL-CSMA/CA throughput vs. number of nodes, $N$, when $T_{u l}=10000$ and $T_{u l}=30000$.

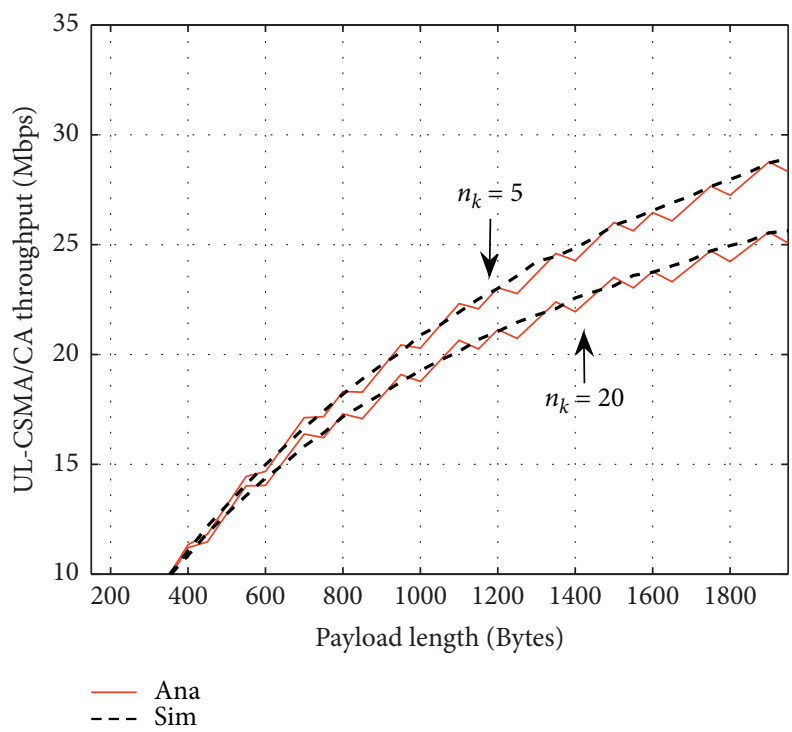

FIGURE 18: UL-CSMA/CA throughput vs. payload length, when $n_{k}=5$ and $n_{k}=20$.

(1) The throughput increases as the payload length increases. The reason is intuitive. A key factor that affects the throughput is how many bytes of frames are transmitted. Therefore, the longer the frame is transmitted, the higher the total throughput is.

(2) The overall throughput when $n_{k}=5$ is higher than that when $n_{k}=20$. The larger the number of nodes, the higher the collision probability in each sector. With collision probability increasing, the throughput is decreased.

Figure 19 plots the throughput of UL-CSMA/CA as the physical data rate varies from $10 \mathrm{Mbps}$ to $100 \mathrm{Mbps}$, where the number of each sector, $n_{k}$, is 5 and 20, respectively. From this figure, we have the following observations.

(1) The simulation curves closely match the corresponding theoretical curves, which manifests that our model is very accurate.

(2) The throughput increases as the physical data rate increases. This is because the higher physical data rate can make more transmissions finished in the same time.

(3) The overall throughput when $n_{k}=5$ is higher than that when $n_{k}=20$. This indicates that sector with a smaller number of nodes usually has a lower collision probability, which in turn leads to a higher throughput. 


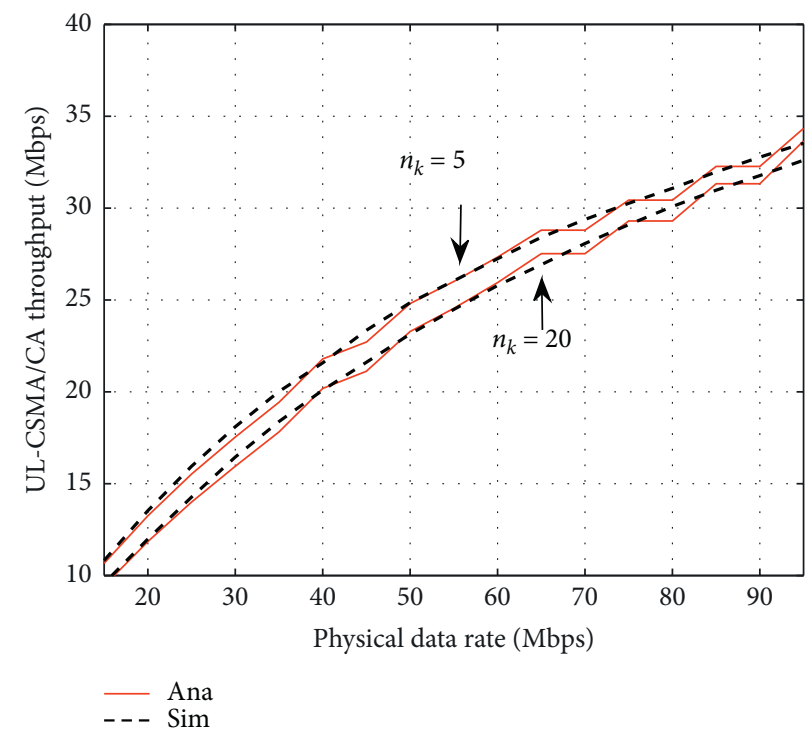

FIgURE 19: UL-CSMA/CA throughput vs. physical data rate, when $n_{k}=5$ and $n_{k}=20$.

TABLE 1: Simulation settings.

\begin{tabular}{lccccc}
\hline$N$ & 100 & $\mathrm{~T}_{\mathrm{PHY} \_ \text {Header }}$ & $20 \mu \mathrm{s}$ & $\mathrm{T}_{\text {SIFS }}$ & $10(\mu \mathrm{s})$ \\
\hline$n_{k}$ & 5,20 & $\mathrm{R}$ & $54 \mathrm{Mbps}$ & $\mathrm{T}_{\text {SLOT }}$ & 20 \\
$K$ & 20,5 & $\mathrm{~L}_{\mathrm{MPDU}}$ & 1528 Bytes & $\mathrm{T}_{\text {DIFS }}$ & 50 \\
$T_{s}$ & $49600 \mu \mathrm{s}$ & $\mathrm{L}_{\text {ACK-Req. }}$ & 14 Bytes & $\mathrm{T}_{\text {ACK-Req. }}$ & 22 \\
$T_{\mathrm{dl}}$ & $19525 \mu \mathrm{s}$ & $\mathrm{L}_{\mathrm{ACK}}$ & 14 Bytes & $\mathrm{T}_{\mathrm{ACK}}$ & 22 \\
$T_{\mathrm{ul}}$ & $30000 \mu \mathrm{s}$ & $\mathrm{L}_{\mathrm{TF}}$ & 124 Bytes & $\mathrm{T}_{\mathrm{TF}}$ & 38 \\
$T_{h}$ & $500 \mu \mathrm{s}$ & $\mathrm{L}_{\mathrm{TF}-\mathrm{END}}$ & 124 Bytes & $\mathrm{T}_{\mathrm{TF}-\mathrm{END}}$ & 38 \\
\hline
\end{tabular}

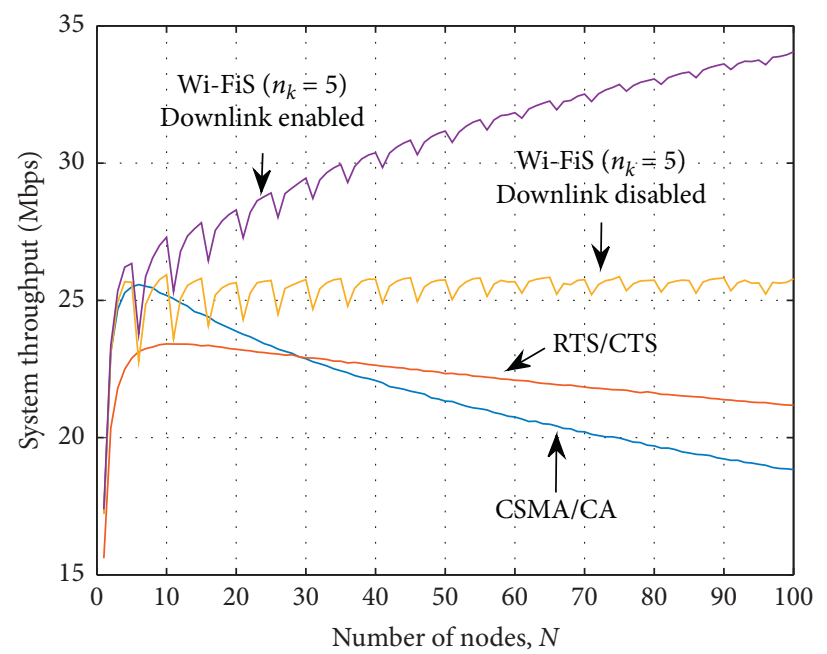

FIgure 20: Comparison of system throughput among Wi-FiS, RTS/CTS, and CSMA/CA.

7.3. Multicell Network. We run simulations for hidden/exposed terminal problems with corresponding settings of Figures 11 and 12, respectively.
Figure 21 plots the throughput of CSMA/CA and Wi-FiS when there are hidden terminals. In this figure, we can see that, for CSMA/CA, hidden terminals cannot monitor each 


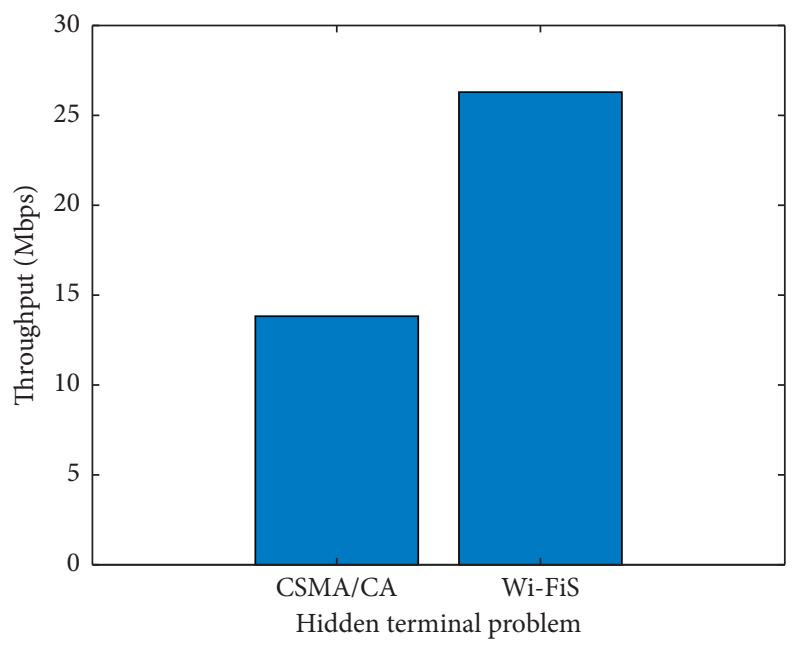

Figure 21: Comparison of throughput between Wi-FiS and CSMA/CA for hidden terminal scenario.

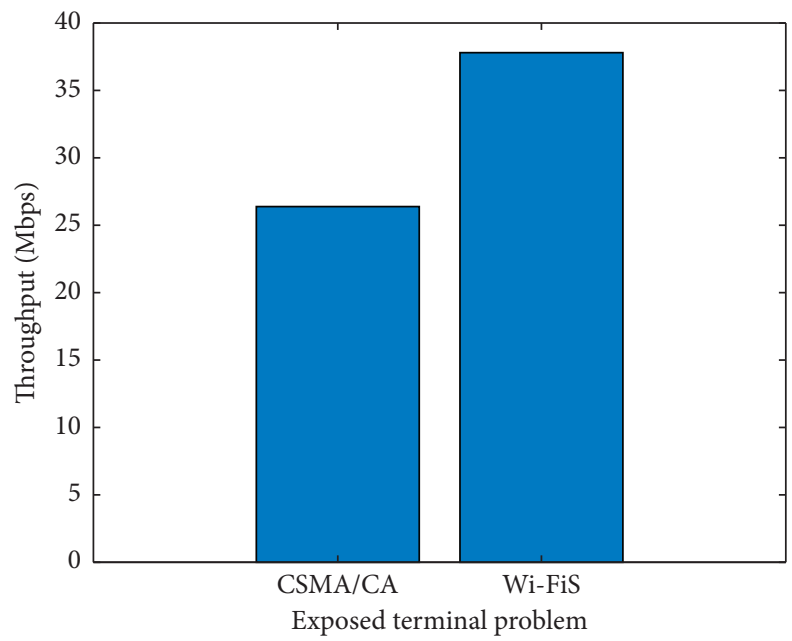

Figure 22: Comparison of throughput between Wi-FiS and CSMA/CA for exposed terminal scenario.

other's existence, so they will transmit at the same time when they cannot transmit data, which will lead to collisions at the receiving node. Therefore, the throughput of CSMA/CA is low. Our protocol uses a partitioned method to allow nodes to perform directional transmission for solving the collision problem caused by hidden terminals, thereby increasing the throughput.

Figure 22 plots the throughput of CSMA/CA and WiFiS when there are exposed terminals. In this figure, we can see that, for CSMA/CA, because the exposed terminals have monitored each other's existence, they may needlessly defer transmitting when they can transmit data, which will lead to channel resource wastage problem, thereby reducing throughput. Therefore, our protocol uses a directional beam to make AP perform downlink transmission to solve the problem of low channel utilization caused by exposed terminals, thereby effectively improving throughput.
In summary, it can be seen that the Wi-FiS is significantly better than the traditional protocol, and as the number of nodes increases, the throughput of Wi-FiS will not fluctuate significantly and is relatively stable. It shows that our protocol has good adaptability in large-scale and high-density Wi-Fi networks, which can ensure overall throughput and improve network performance.

\section{Conclusions}

$5 \mathrm{G}-\mathrm{CPE}$ integrates $5 \mathrm{G}$ and $\mathrm{Wi}-\mathrm{Fi}$ protocols and is one of the key technologies that bring $5 \mathrm{G}$ to Industry 4.0. In order to solve the collision and hidden/exposed problems in largescale 5G-CPE Wi-Fi networks, this paper designs a new wireless access protocol called Wi-FiS. Wi-FiS divides a dense network into different sectors and performs sector scheduling and therefore significantly reduces transmission 
collision. For multicell networks, utilizing directional beam, Wi-FiS can avoid hidden and exposed terminal problems, while enabling parallel transmissions among multiple cells. A salient feature of Wi-FiS is that it effectively utilizes (rather than modifying) the virtual carrier sense mechanism of conventional Wi-Fi protocols for sector scheduling and therefore has good compatibility with conventional Wi-Fi protocols. We then theoretically analyze Wi-FiS's throughput. Extensive simulations show that the proposed model is very accurate, and the proposed design greatly outperforms related designs and can make a dense network achieve high throughput.

\section{Data Availability}

The data used to support the findings of this study are available from the corresponding author upon request.

\section{Conflicts of Interest}

The authors declare that there are no conflicts of interest regarding the publication of this paper.

\section{Acknowledgments}

This studywas funded in part by the National Natural Science Foundation of China (61872451 and 61872452), in part by the Science and Technology Development Fund, Macau SAR (0098/2018/A3, 0076/2019/A2, and 0037/2020/A1), and in part by the Dongguan Social Science and Technology Development (General) Project (2020507154645).

\section{References}

[1] International Telecommunications Union Radio Communication Sector (ITU-R), "Minimum requirements related to technical performance for IMT-2020 radio interface(s)," Report ITU-R M.2410-0 (11/2017), International Telecommunications Union Radio Communication Sector (ITU-R), Geneva, Switzerland, 2017, https://www.itu.int/\%20pub/RREP-M.2410-2017.

[2] 5G Alliance for Connected Industries and Automation (5G ACIA), "5G for connected industries and automation white paper," 2019, https://www.5g-acia.org/fileadmin/5G-ACIA/ Publikationen/Whitepaper_5G_for_Connected_Industries_ and_Automation/WP_5G_for_Connected_Industries_and_ Automation_Download_19.03.19.pdf.

[3] M. Simsek, A. Aijaz, M. Dohler, J. Sachs, and G. Fettweis, "5Genabled tactile internet," IEEE Journal on Selected Areas in Communications, vol. 34, no. 3, pp. 460-473, 2016.

[4] S. Sen, R. R. Choudhury, and S. Nelakuditi, "CSMA/CN: carrier sense multiple access with collision notification," in Proceedings of the ACM 16th Annual International Conference on Mobile Computing and Networking, pp. 25-36, Chicago, IL, USA, September 2010.

[5] S. Gollakota and D. Katabi, "Zigzag decoding," ACM SIGCOMM Computer Communication Review, vol. 38, no. 4, pp. 159-170, 2008.

[6] T. Xiong, J. Zhang, J. Yao, and W. Lou, "Symbol-level detection: a new approach to silencing hidden terminals," in Proceedings of the 2006 IEEE International Conference on Network Protocols, pp. 1-10, Austin, TX, USA, October 2012.
[7] E. Magistretti, O. Gurewitz, and E. W. Knightly, "802.11ec: collision avoidance without control messages," in Proceedings of the 18th annual international conference on Mobile computing and networking, pp. 65-76, Istanbul, Turkey, August 2012.

[8] A. Acharya, A. Misra, and S. Bansal, "Design and analysis of a cooperative medium access scheme for wireless mesh networks," in Proceedings of the 2004 IEEE International Conference on Broadband Networks, San Jose, CA, USA, October 2004.

[9] T. Yamaguchi, K. Shishida, H. Hirai, K. Tsuneta, and M. Sato, "Improvement of servo robustness for digital sector servo system (hard disk drives)," IEEE Transactions on Magnetics, vol. 28, no. 5, pp. 2910-2912, 1992.

[10] N. Shahin, R. Ali, S. W. Kim, and Y.-T. Kim, "Cognitive backoff mechanism for IEEE802.11ax high-efficiency WLANs," Journal of Communications and Networks, vol. 21, no. 2, pp. 158-167, 2019.

[11] D.-J. Deng, C.-H. Ke, H.-H. Chen, and Y.-M. Huang, "Contention window optimization for IEEE 802.11 DCF access control," IEEE Transactions on Wireless Communications, vol. 7, no. 12, pp. 5129-5135, 2008.

[12] R. Ali, N. Shahin, Y. B. Zikria, B.-S. Kim, and S. W. Kim, "Deep reinforcement learning paradigm for performance optimization of channel observation-based MAC protocols in dense WLANs,” IEEE Access, vol. 7, pp. 3500-3511, 2018.

[13] L. Wang, K. Wu, and M. Hamdi, "Attached-RTS: eliminating an exposed terminal problem in wireless networks," IEEE Transactions on Parallel and Distributed Systems, vol. 24, no. 7, pp. 1289-1299, 2012.

[14] Y.-H. Lee and W.-R. Wu, "A WLAN uplink collision-resolving scheme using multi-user beamforming technique," IEEE Transactions on Vehicular Technology, vol. 69, no. 10, pp. 11042-11054, 2020.

[15] IEEE 802.11, "Wireless LAN medium access control (MAC) and physical layer (PHY) specifications," ANSI/IEEE Std 802.11, 2016.

[16] A. Gaber and A. Omar, "A study of wireless indoor positioning based on joint TDOA and DOA estimation using 2-D matrix pencil algorithms and IEEE 802.11ac," IEEE Transactions on Wireless Communications, vol. 14, no. 5, pp. 2440-2454, 2015.

[17] A. Neri, A. Di Nepi, and A. M. Vegni, "DOA and TOA based localization services protocol in IEEE 802.11 networks," Wireless Personal Communications, vol. 54, no. 1, pp. 155-168, 2010.

[18] F. Wen and C. Liang, "An indoor AOA estimation algorithm for IEEE 802.11ac Wi-Fi signal using single access point 2014.11ac Wi-Fi signal using single access point," IEEE Communications Letters, vol. 18, no. 12, pp. 2197-2200, 2014.

[19] IEEE 802.11-TGe, "Wireless LAN medium access control (MAC) and physical layer (PHY) specifications, specificationamendment 8 :meduim access control (MAC) quality of service enhancements," ANSI/IEEE Std 802.11, 2005.

[20] N. M. Sabah and A. Hocanin, "The use of negative acknowledgement control packets (NACKs) to improve throughput and delay in IEEE 802.11 networks," in Proceedings of the 2010 2nd International Conference on Computer Technology and Development, pp. 136-140, Cairo, Egypt, November 2010.

[21] L. Zheng, M. Ni, L. Cai, J. Pan, C. Ghosh, and K. Doppler, "Performance analysis of group-synchronized DCF for dense IEEE 802.11 networks," IEEE Transactions on Wireless Communications, vol. 13, no. 11, pp. 6180-6192, 2014. 